Chromosoma (Berl.) 16, 415-435 (1965)

From the Cytogenetics Laboratory, Carnegie Institution of Washington, and Departments of Botany and Zoology,

The University of Michigan, Ann Arbor, Michigan

\title{
NUMBER AND PATTERN OF ASSOCIATION \\ OF CHROMONEMATA IN THE CHROMOSOMES OF TRADESCANTIA*
}

\author{
By
}

Elio Sparvoli, Helen Gay, and Berwind P. Kaufmann

With 13 Figures in the Text

(Received September 27, 1964)

\section{Introduction}

Studies of the patterns of incorporation and distribution of tritiated thymidine within the chromosomes of higher organisms (e.g., TAYLoR, Woods, and HugHes 1957; TAYLoR 1958) have led to the interpretation that the mitotic-type chromosome is composed of a single chain of DNA molecules before interphasic replication (TAYLoR 1958, $1959,1962,1963)$. Although other studies employing similar experimental procedures have given results that led to different interpretations (La Cour and Pelc 1958; Peacock 1963), many questions have been raised in the past few years about the validity of the observational evidence (summarized by KaUfManN, GaY and MoDonald 1960; RIs 196I) from which the premise has been derived that the chromosomes of higher plants and animals are multistranded.

To throw new light on these basic problems of chromosome organization, we have prepared and studied three-dimensional reconstructions of serial sections of chromosomes of Tradescantia paludosa during different stages of the mitotic cycle. Our observations indicate that the chromosomes are indeed multistranded in terms of the number of chromonemata (using the definition of NEBEL 1939, that a chromonema is one of the "optically single threads within the chromosome").

* This paper is dedicated to Professor Hans Bader on his sixtieth birthday anniversary in appreciation of his contributions to the development of modern cytology.

The work reported here was supported in part by Research Grants GM-10499 from the National Institutes of Health, U.S. Public Health Service, and GB-290 from the National Science Foundation, and in part by a NATO fellowship awarded to E. Sparvoli by the Italian National Council of Research. 


\section{Materials and Methods}

The report given here is based on a study of the apical cells of staminate hairs of T. paludosa. This material was chosen for the following reasons: (a) problems of fixation are lessened (as compared with those encountered when handling aggregates of cells, such as occur in root tips) because penetration of reagents is facilitated by the arrangement of cells end to end in single file; (b) the apical cells of the hairs are actively engaged in division; (c) cells in any desired phase of mitosis can be selected for critical examination by rapid scanning procedures; (d) cells chosen for study can be oriented when being embedded so that they can be sectioned in any desired plane.

Staminate hairs having terminal cells actively engaged in mitosis are encountered most frequently on the filaments of those stamens whose anthers have a pale yellow color. Buds of the appropriate age were opened, so that the intact stamens could be removed one by one and transferred to the fixative. This was a $1 \%$ solution of

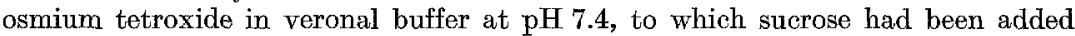
in the amount of $15 \mathrm{mg}$ per ml, as specified by CAULFIELD (1957). Immediately after placing the tissues in the fixative, the containers were transferred to a vacuum desiccator connected with an aspirator for removal of air that was trapped around the staminate hairs. Within 10 minutes or so the air had been removed and the tissue had sunk to the bottom of the container; aspiration was then terminated but the period of fixation was continued for about two hours.

After thorough washing in distilled water, the material was treated for half an hour in a saturated aqueous solution of uranyl acetate. An additional rinse in distilled water was followed by dehydration. For this purpose a carefully graded series of distilled water-ethanol mixtures was used (beginning with $1 \%$ ethanol, and progressing through $2,5,7,10,15,20,25 \cdots 90,95$, to absolute alcohol) in order to minimize the possibility of causing marked distortion of the contents of the staminate-hair cells. The dehydrated tissues were then transferred, preparatory to infiltration, through a series of increasing concentrations of propylene oxide in absolute ethanol until they reached pure propylene oxide.

Infiltration with the embedding medium-Maraglas, mixture $\mathrm{E}$, with added accelerator, as described by FremMan and SPURLOck, 1962-was effected by gradually increasing the concentration of the resin in propylene oxide, starting with $0.5 \%$, and passing through $1,2,4,8,10,15,20,30 \cdots 90 \%$ before reaching the resin alone. At this stage the individual stamens lying in drops of resin were examined under the dissecting microscope, and the hairs were detached one by one, using fine tungsten needles that had been sharpened in fused sodium nitrite.

Those hairs that seemed suitable for further study were then transferred to small droplets of resin lying on a microscope slide that had previously been coated with a thick layer of evaporated carbon. The slides--usually containing two rows of six or seven droplets-were placed in the oven where they remained for about 72 hours to permit polymerization of the resin. After that, each of the dividing staminate-hair cells was examined with the compound microscope to identify accurately the mitotic phase represented. Capsules filled with resin were then inverted over those laminae that contained cells which were to be sectioned. Subsequently, the slide was returned to the oven for final "curing" of the resin. When hardened, the block (enveloped in a gelatin eapsule) was removed from the slide, the thick carbon layer serving effectively as a parting face. It should be added that if sectioning of chromosomes in a given plane is desired, the cells can be properly oriented by positioning the hair with fine-pointed tungsten needles in the drop of resin before it is completely polymerized. Additional details of this open-face method of embedding are given in another publication (Sparvoli, GAY, and KAUTMANN 1965). 


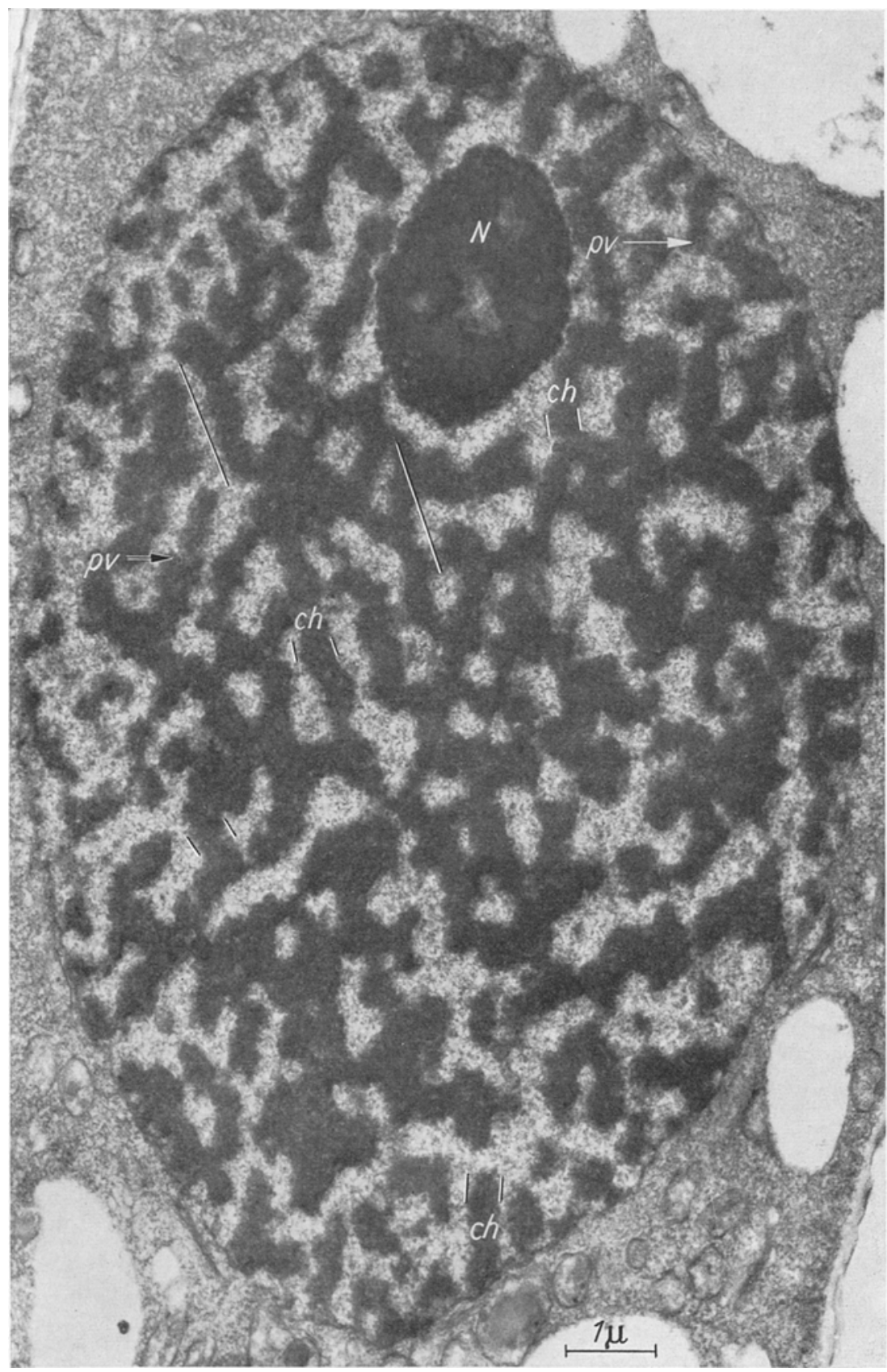

Fig. 1. Nucleus in preprophase. The nucleus appears as a network of chromonemata $(c h), 0.3 \mu$ thick where they lie stretched and free from entanglement (for example between the double lines). Note that the chromonemata tend to run parallel to the major axis of the nueleus (e.g., the longer lines). The nucleolus $(N)$ is free from the adjacent chromonemata. $(p v)$ perichromatin vesicles. $\times 11,250$ 
Serial sections were prepared with an L.K.B. Utratome. The sections were picked up with a wire loop and transferred to a petri dish, from which they were collected on a formvar membrane supported by a wire loop for final transfer to either a Sjöstrand- or Anderson-type grid by the method of GAY and ANDERson (1954). Electron micrographs of the serial sections were obtained with an RCA EVU-3 G electron microscope.

For three-dimensional reconstruction, the outlines and other salient topographical features of each consecutive section were traced on a thin sheet of dental wax (base plate or bite wax). When cut out and stacked, these thin sheets provided reconstructions of chromosomes in which the relationships of individual chromonemata could be determined. The interpretations given below are based on studies of several reconstructions, some of which involved 20 or more consecutive longitudinal serial sections through a single chromosome.

\section{Results}

In this paper we shall consider the chromonema as a structural entity without attempting to define its internal or subchromonematic organization.

\section{Diameter of the chromonema}

The chromonemata appear in our preparations as clearly separable strands during prophase and telophase (Figs. $1-3,9-11$ ), whereas they are less readily discernible as individual entities during interphase, metaphase, and anaphase (Figs. 7, 8, 12, 13).

The diameter of the chromonema, when it first becomes conspicuous during pre-prophase in favorable, wellstretched preparations is about $0.3 \mu$ (Fig. 1). In late prophase the diameter has decreased to about $0.25 \mu$ (Fig. 3), and at early telophase it measures only $0.2 \mu$ (Fig. 9). At the end of telophase it increases again to 0.25 or $0.3 \mu$ (Figs. 10, 11).

It has not been possible to obtain any precise measurement of the length of a single chromonema, although on a comparative basis it would seem from our reconstructions that the maximum length is attained during pre-prophase and that there is a tendency for a decrease to occur during prophase and telophase.

\section{Patterns of association and number of chromonemata}

The individual chromosomes are not clearly delimited in the preprophase nucleus, so that the constituent chromonemata appear to form a three-dimensional network. But it is obvious from Fig. 1 that the strands are oriented for the most part parallel to the longer axis of the nucleus. Reconstructions from serial sections suggest, moreover, that the chromonemata do not anastomose freely, although they may lie in close proximity to one another.

As prophase advances, the distinction between individual chromosomes of the complex becomes more pronounced, largely because groups 


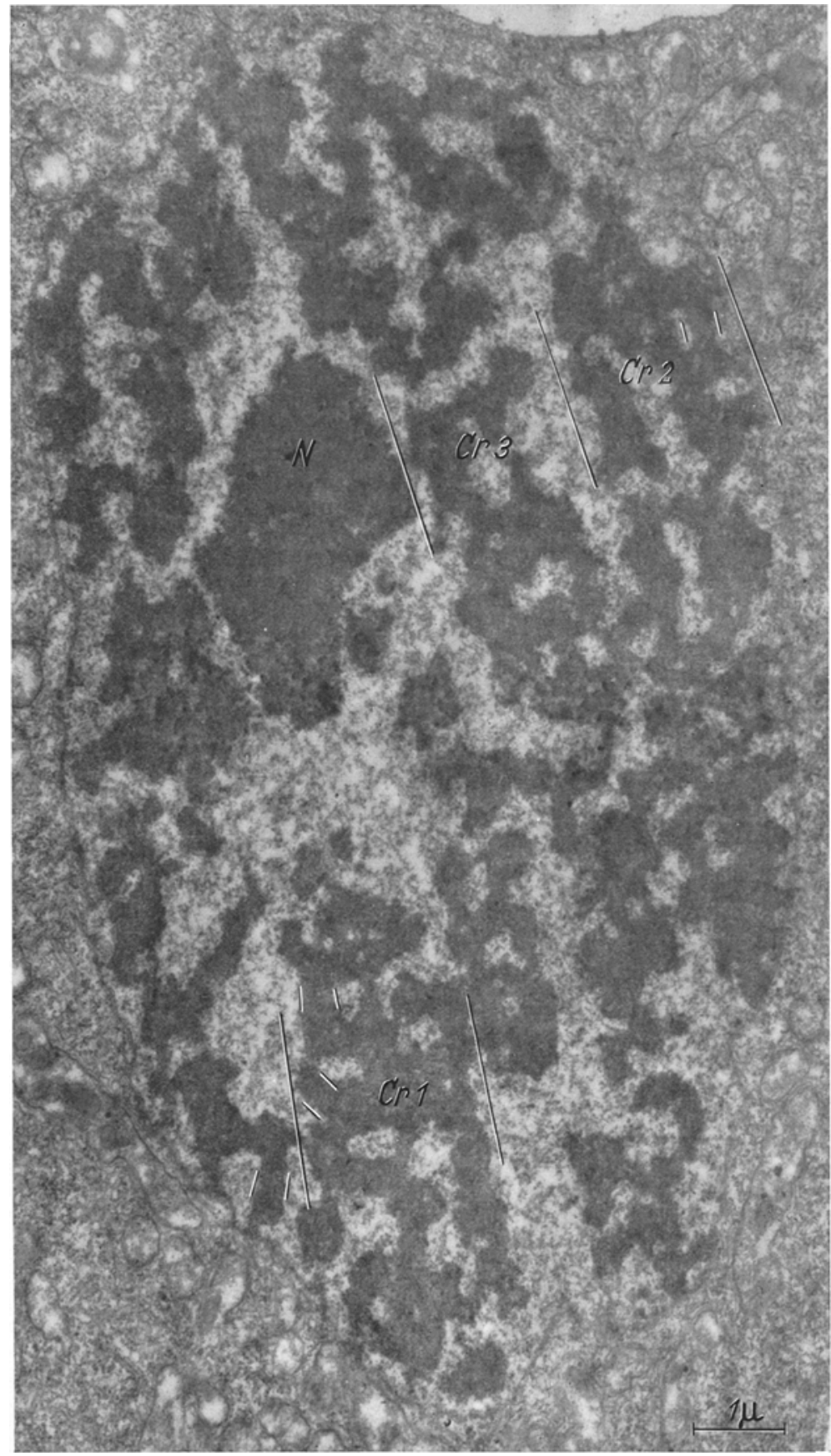

Fig. 2. Nucleus in early prophase. The chromonemata are associated in Iongitudinal bundles to form the chromosomes $(C r)$ which are separated by narrow spaces of nucleoplasm. The chromosome $\mathrm{Cr} 1$ is seen in a frontal view (compare with the model in Fig. 5, T). The chromosomes $\mathrm{Cr} 2$ and $\mathrm{Cr} 3$ are seen in a side view (compare with the model in Fig. 5, 0). Where two or more chromonemata lie in close contact their morphological distinction in the micrographs is no longer evident. $(N)$ nucleolus. $\times 11,250$ 


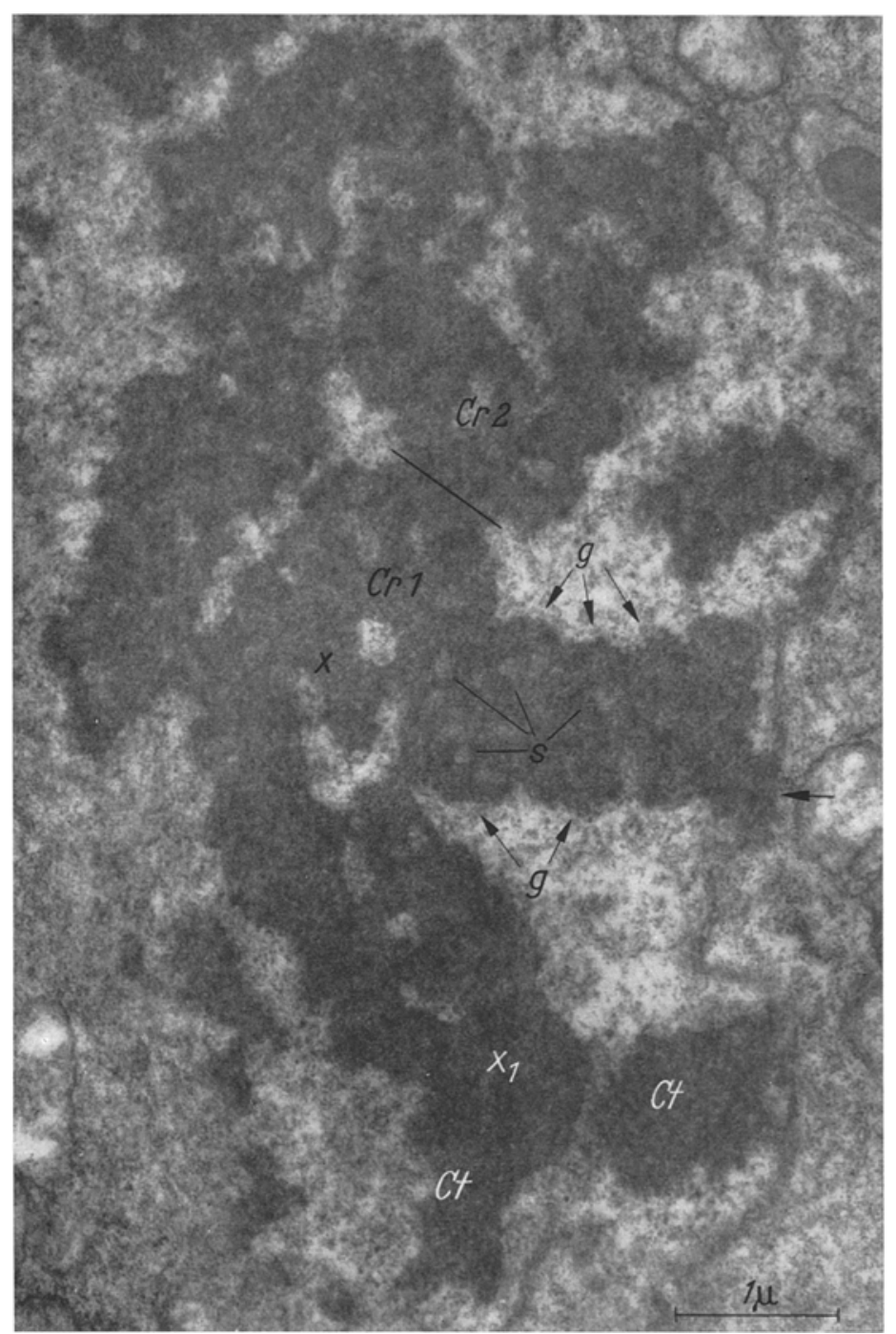

Fig. 3. Nucleus in late prophase. The chromonemata are now closely associated and show only a few visible spaces within the chromosorne (s). Nevertheless it is still possible to recognize their typical association in the diplospireme in the horizontal extension of chromosome $C \% 1$. This end abuts on the nuclear membrane with the tip of one chromonema shown by an arrow. The opposite end shows a division into two chromatids $(C t)$ which also abut on the nuclear membrane. $X$ and $X_{1}$, represent twists of the chromosome. $(g)$ gyres of the diplospireme. $\times 17,500$

of chromonemata appear as aggregates which are separated from other similar groups by spaces filled with nucleoplasm (Fig. 2). It thus becomes possible at this stage to determine the patterns of association of the chromonematic sub-units that form the chromatids and chromosome. 

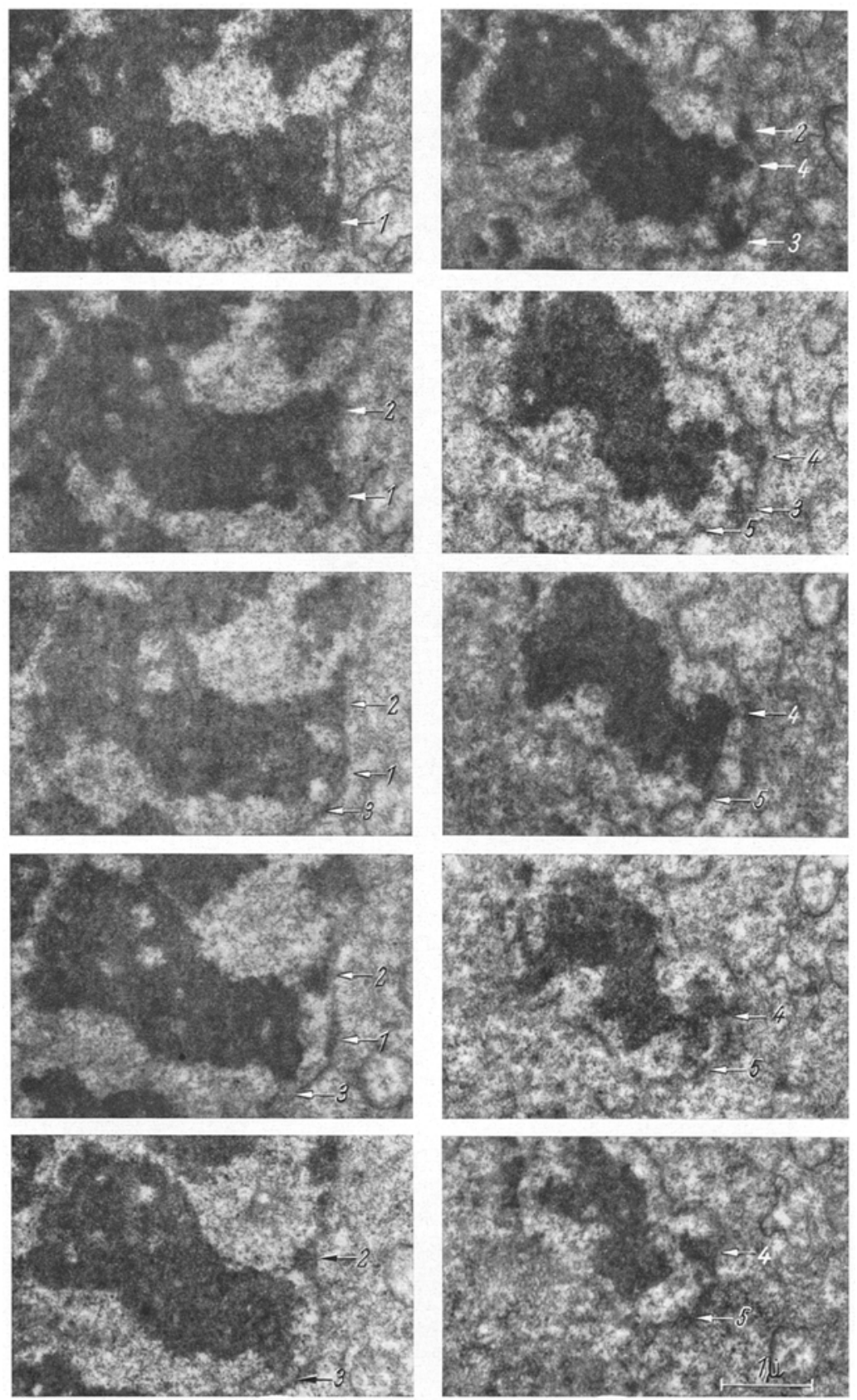

Fig. 4. Late prophase chromosome (same as $\mathrm{Cr} 1$ of Fig. 3). The ten consecutive sections show five chromonematic terminations at the nuclear membrane. $\times 11,250$ 
Ultrathin sections of favorably oriented stretches of prophase chromosomes reveal densely-electron-scattering materials in the form of a series

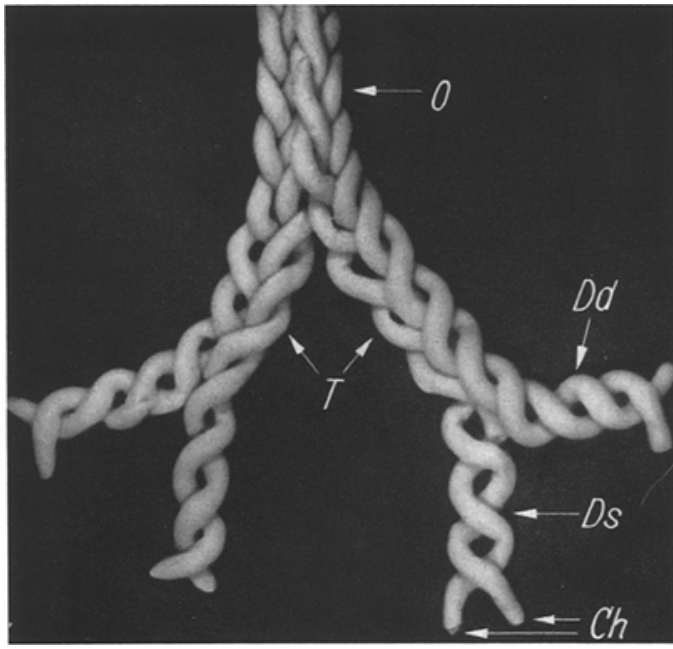

Fig. 5. Model of a prophase chromosome. Two chromonemata $(C h)$ associate to form a diplospireme $(D s, D d)$. Sinistrorse and dextrorse diplospiremes $(D s$ and $D d$ ) associate and superimpose laterally to form a tetraspireme $(T)$, which is equivalent to a chromatid. Two tetraspiremes form an "octospireme" (O), which is seen here in side view; this corresponds to the prophase chromosome

waces in the models prepared by stacking wax sheets represen ting individual serial sections (Fig. 6). of links or rings (Figs. 2, 3). The general over-all impression gained from inspection of such sections is that the chromosome is composed of helically disposed chromonemata. Reconstructions, prepared from consecutive serial sections, indicate that the ringlike appearance results from a complex, but precise, aggregation of several chromonematic subunits. The criteria used in reaching that interpretation, which is specified below, include analysis of the relationship of marginal contours, internal sinuses, and open

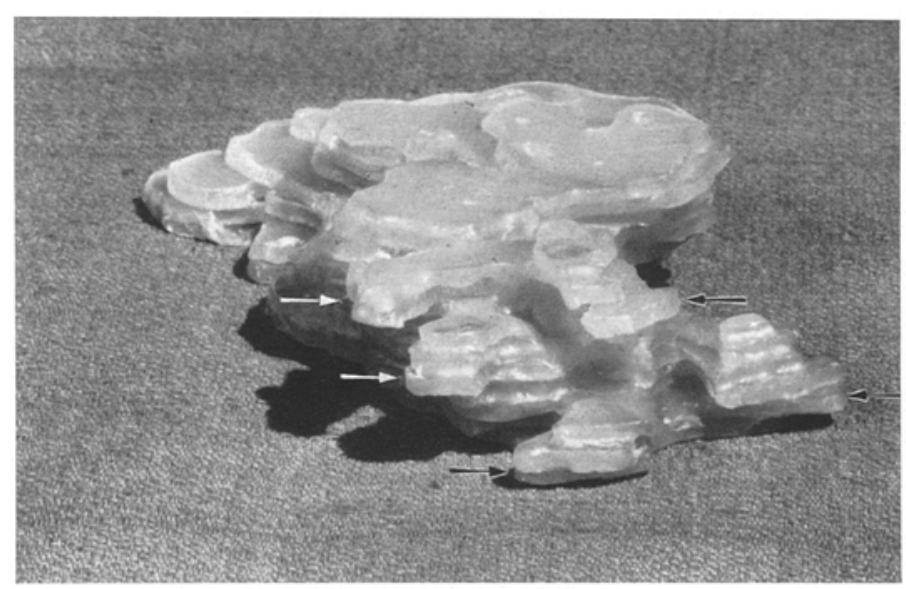

Fig. 6. Partial reconstruction of a chromosome obtained by stacking 10 sheets of wax (representing the 10 successive sections shown in Fig. 4), which were cut out to show the contours and ends of the component chromonemata 


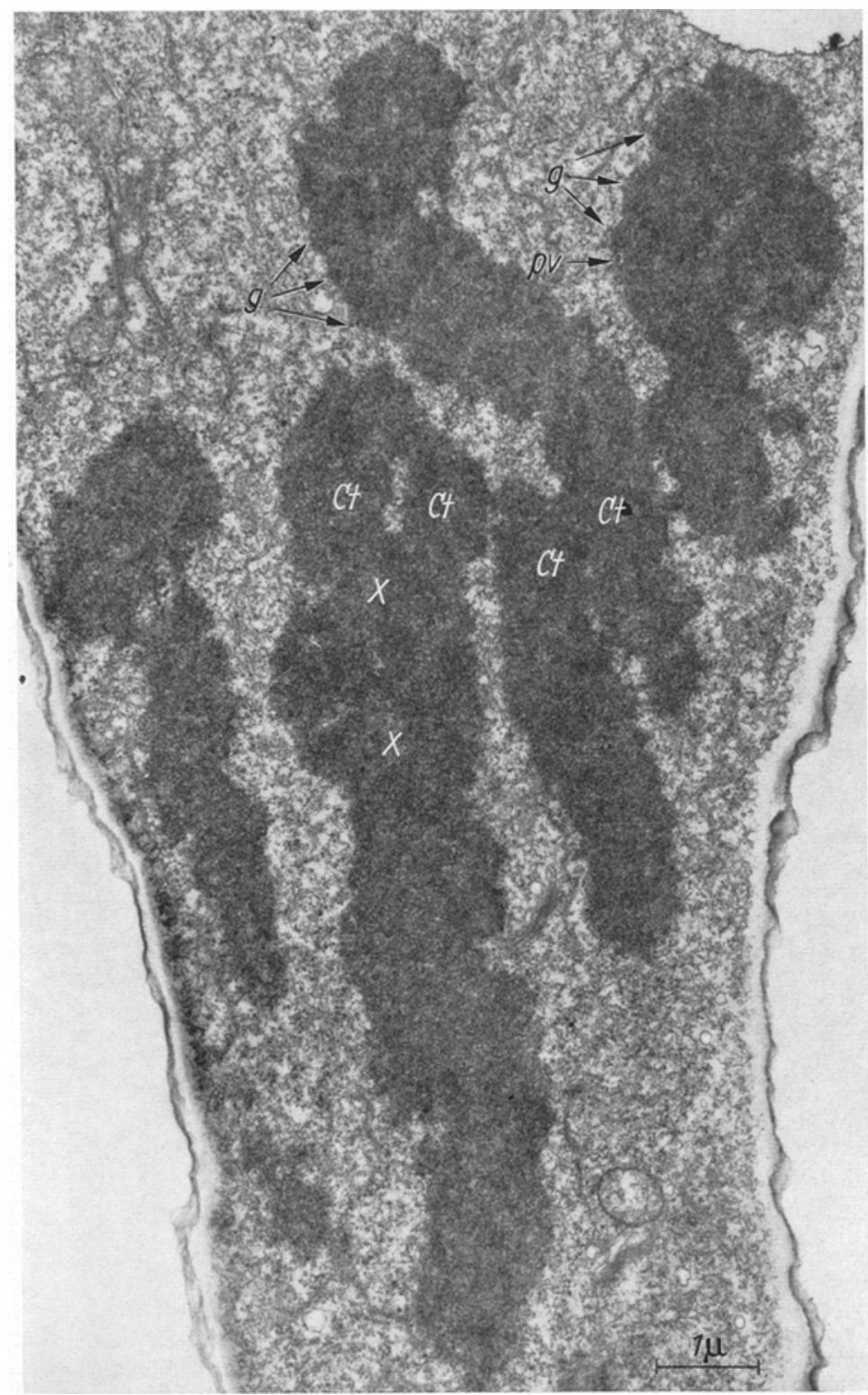

Fig. 7. Metaphase chromosomes. Although the chromonemata are no longer distinguishable as morphologically identifiable threads, their presence is suggested by the sinuosity of the contours of the chromosome $(g)$. $(x)$ twists of the chromatids $(C t)$ or relational coilling. $(p v)$ perichromatin vesicle. $\times 11,250$

According to our interpretation, the fundamental structural unit is a pair of helically arranged chromonematic strands (Fig. 5). The two strands of this unit assumedly can be arranged in either a dextrorse 
424 E. Sparvoli el al.: Number and association of chromonemata in Tradescantia

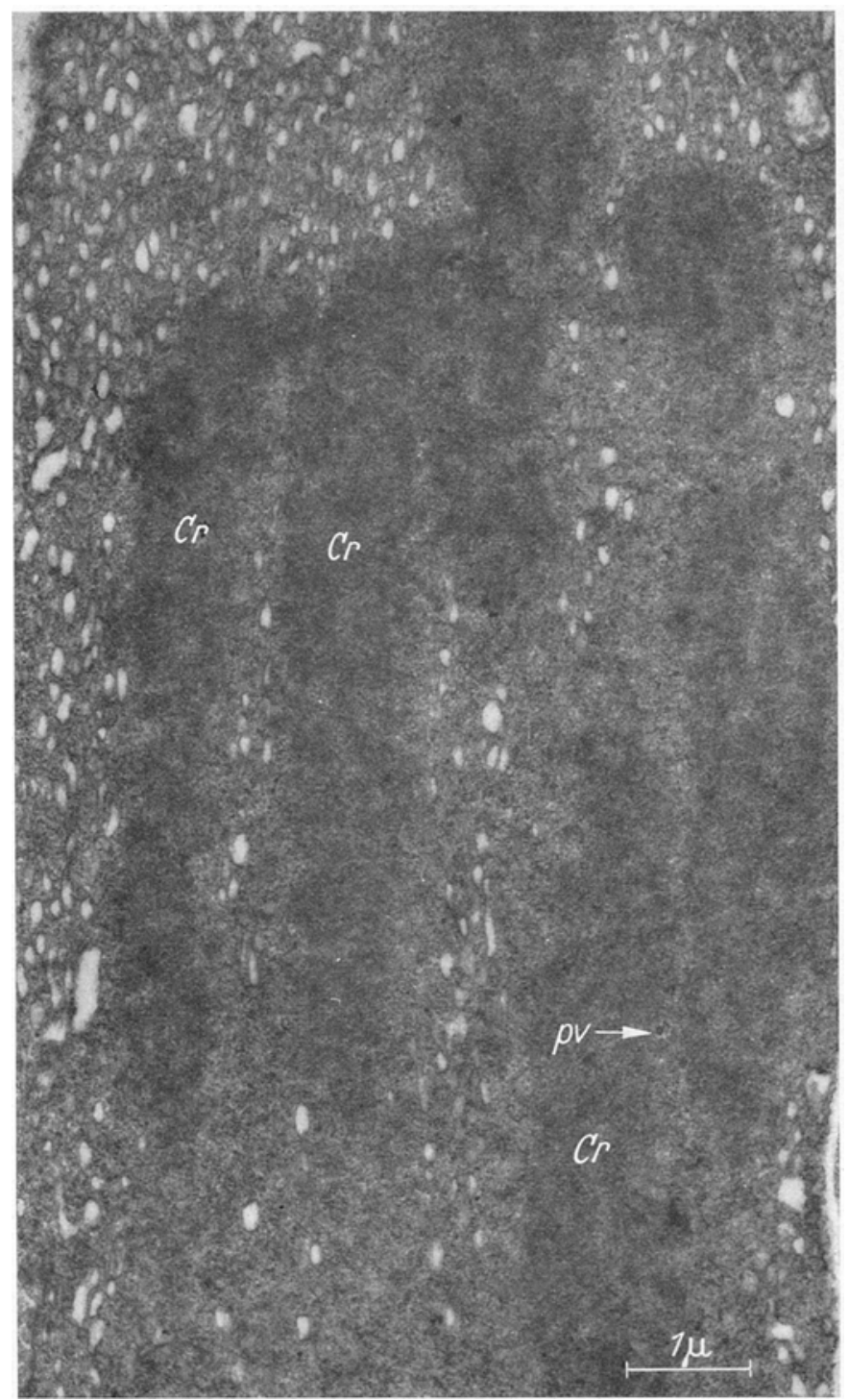

Fig. 8. Late anaphase chromosomes. The chromosomes are surrounded by and in continuity with a dense mantle containing amorphous substances, fibcillar materials and a large number of ribosomes. The cy toplasm is also dense and shows numerous vesieies some of which are aligned along the chromosomes, $(p v)$ perichromatin vesicle. $\times 13,500$

or a sinistrorse relationship. If isolated, the unit would appear chainlike in face view but flat or ribbon-like in side view. In other words, the two paired chromonemata lie in close association at the points of overlap but are more widely separated in the intervening loop regions. We shall call such a double helix a "diplospireme". 


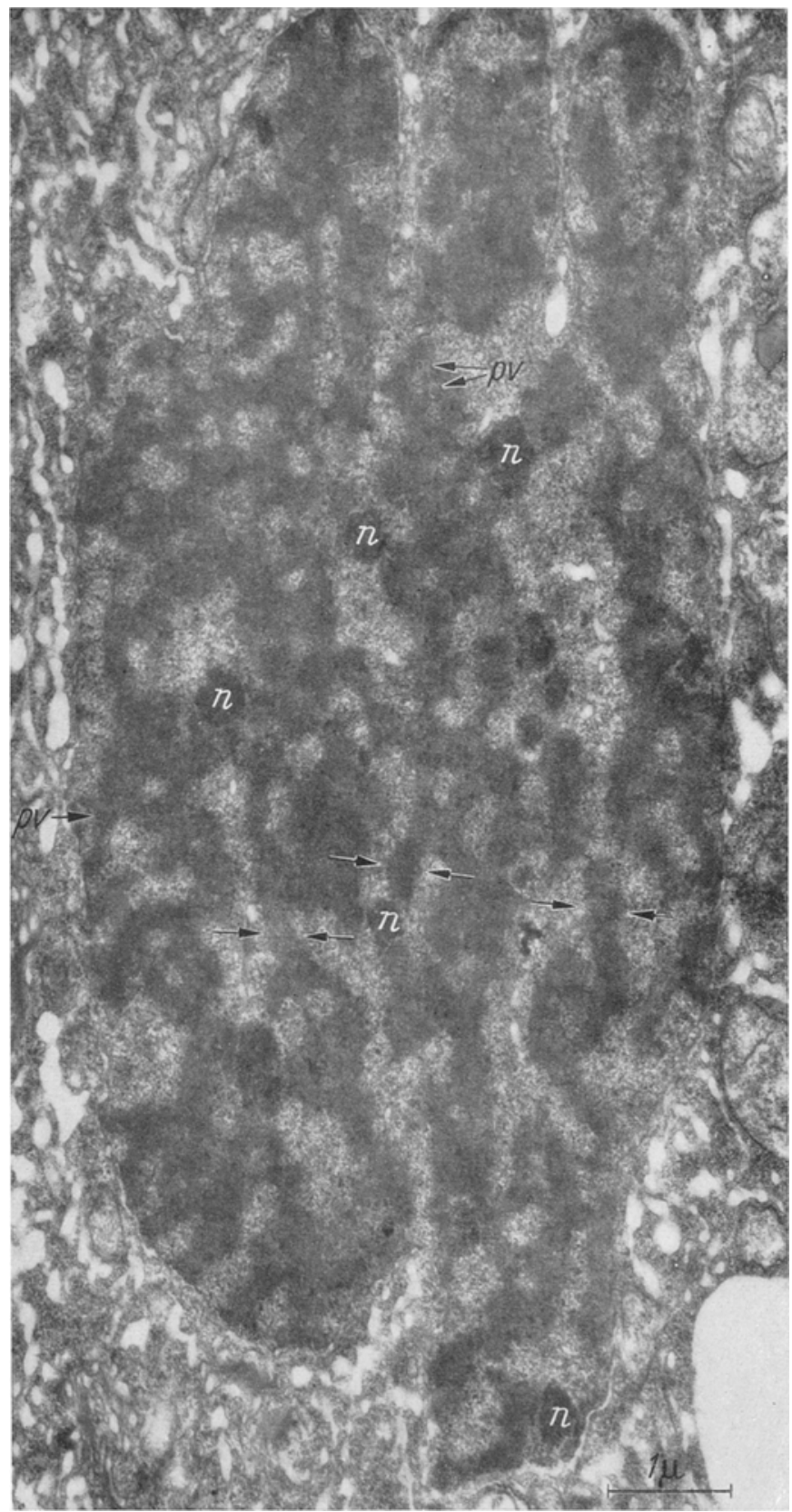

Fig. 9. Nucleus in early telophase. A continuous nuclear membrane has just been laid down around the nucleus. It often appears swollen, suggesting its origin from coalescence of those vesicles that surrounded the group of chromosomes in late anaphase. On the other hand, the vesicles in the cytoplasm have fused to form sausage-shaped lacunae. The chromonemata appear again as distinguishable strands (e.g., between arrows) surrounded by the dense nuclear matrix. Pronucleoli $(n)$ originate by the aggregation of ribosome-like granules as well as fibrillar and amorphous materials, usually in the spaces between chromosomes but with no other preferential location; $(p v)$ perichromatin vesicles. $\times 14,000$ 
It appears, moreover, that pairs of diplospiremes are associated to form a higher unit in the structural hierarchy that we shall call a "tetraspireme". Our analysis suggests that dextrorse and sinistrorse diplo-

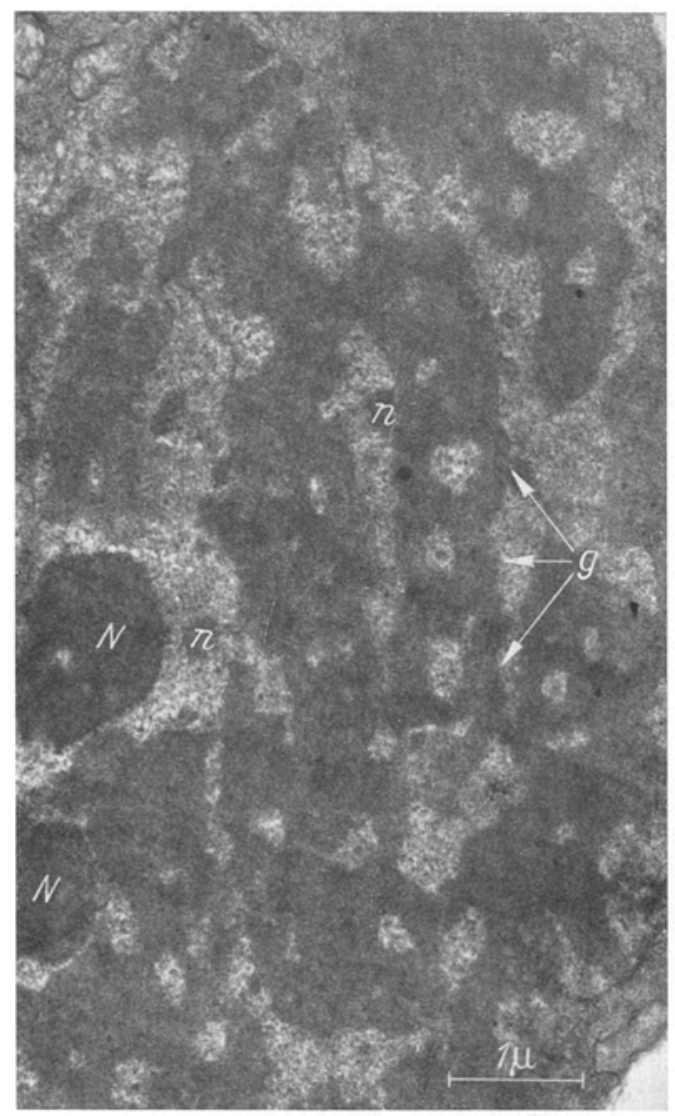

Fig. 10. Nucleus in middle telophase. The chromonemata are clearly distinguishable. Note their association to form the basic diplospireme $(g)$. ( $N$ ) nucleoli, possibly formed by the fusion of pronucleoli $(n) . \times 13,500$ spiremes associate to form these twin complexes. If dextrorse associated with dextrorse, or sinistrorse with sinistrorse, contour and spacing patterns should be established which differ from those observed. Reconstructions derived from serial sections of the two chromosomes labeled $\operatorname{Cr} 1$ in Figs. 2 and 3 suggest, moreover, that associa. tion of diplospiremes in volves not only apposition but also superposition, in the manner depicted in Fig. 5. This interpretation stems from the observation that the twin structure frequently lacks the width that would be expected if two diplospiremes were lying side by side, but has the smaller diameter that would be expected if the two complexes overlapped at their inner

borders. Viewed from another angle, the twins could appear as a single unit due to superposition of one diplospireme with respect to the other (Fig. 5).

Additional evidence suggests that the tetra- spireme represents an intermediate structural level in the organizational hierarchy of the chromosome, the entire body being an "octospireme" when defined in terms of the number of chromonemata (Fig. 5). One type of evidence is afforded by the chromosome labeled $\operatorname{Cr} 1$ in Fig. 3. Here the tip of a late 
prophase chromosome abuts on the nuclear membrane. This chromosome runs straight for a short distance before it turns and twists, but it can be followed to its other end, where it is clearly separated into two

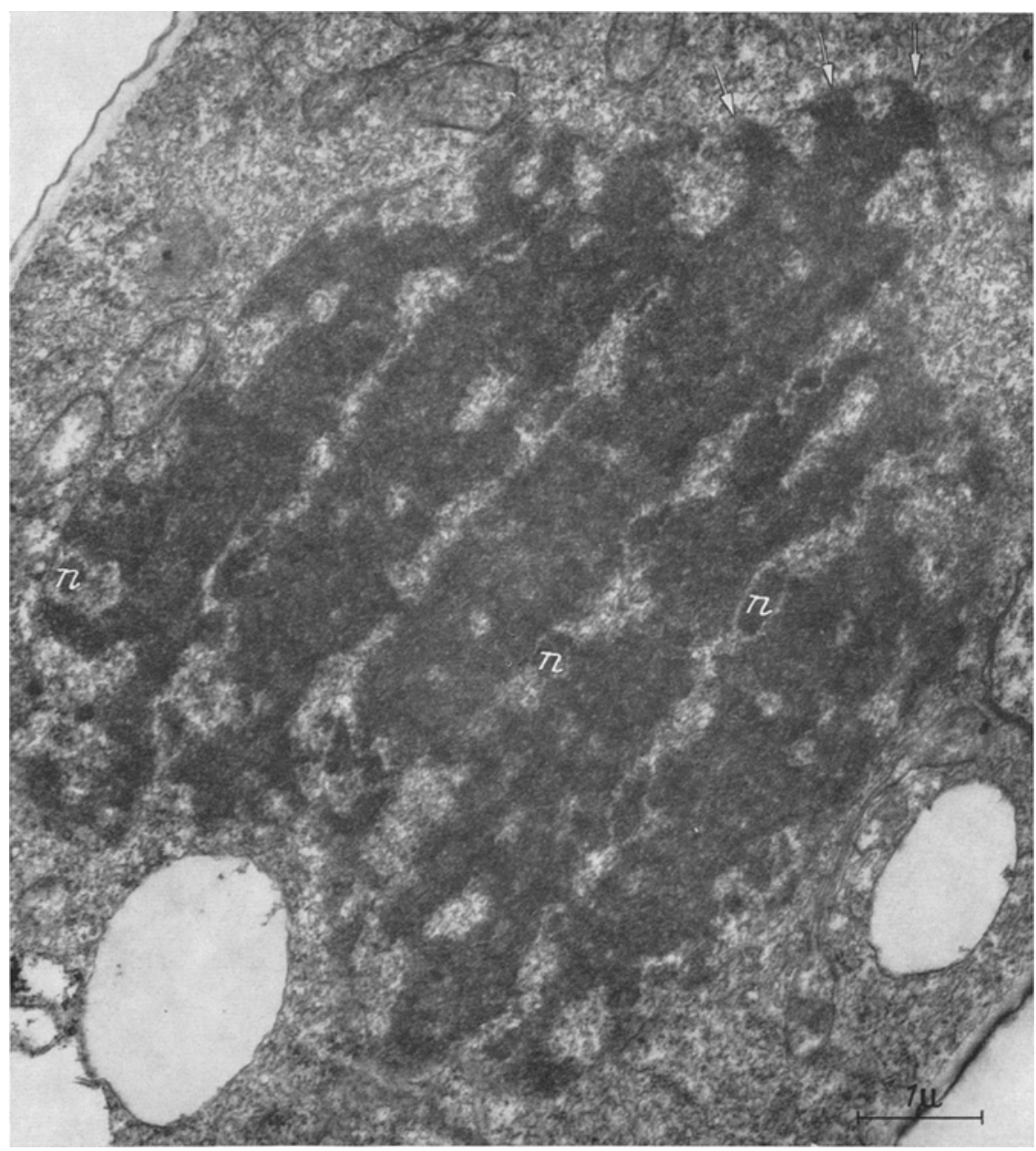

Fig. 11. Nucleus in late telophase. The chromonemata and their manner of association are no longer clearly discernible, although in some cases (e.g., the chromosome lying in the middle of the nucleus) it is possible to see their termination at the nuclear membrane (arrows). (n) pronucleoli. $\times 13,500$

chromatids that also lie in contact with the nuclear membrane. A three-dimensional model made from ten consecutive serial sections through the straight stretch of this chromosome showed five chromonematie ends in contact with the nuclear membrane (Fig. 6). Comparison of successive sections (Fig. 4) afforded evidence, moreover, that these were free ends rather than folds or loops in the chromonematic strands 
428 E. Sparvoli et al.: Number and association of chromonemata in Tradescantia

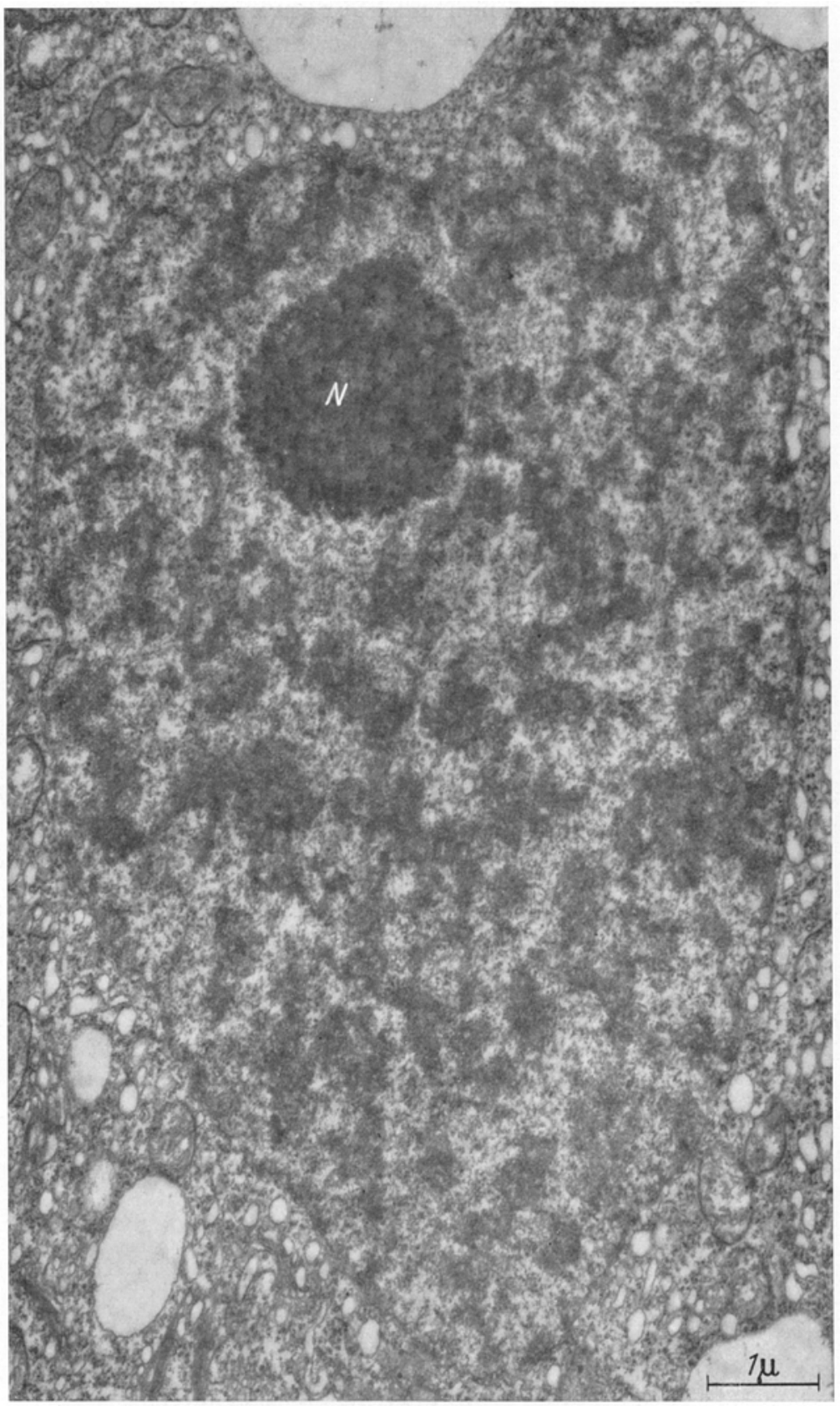

Fig. 12. Nucleus in early interphase. The nucleus is pear shaped as it commonly appears in late telophase (e.g., Fig. I1). The chromonematic material, although aggregated in clumps in some places, is largely unravelled and diffused in the nucleoplasm. $(N)$ nucleolus. $\times 13,500$ 


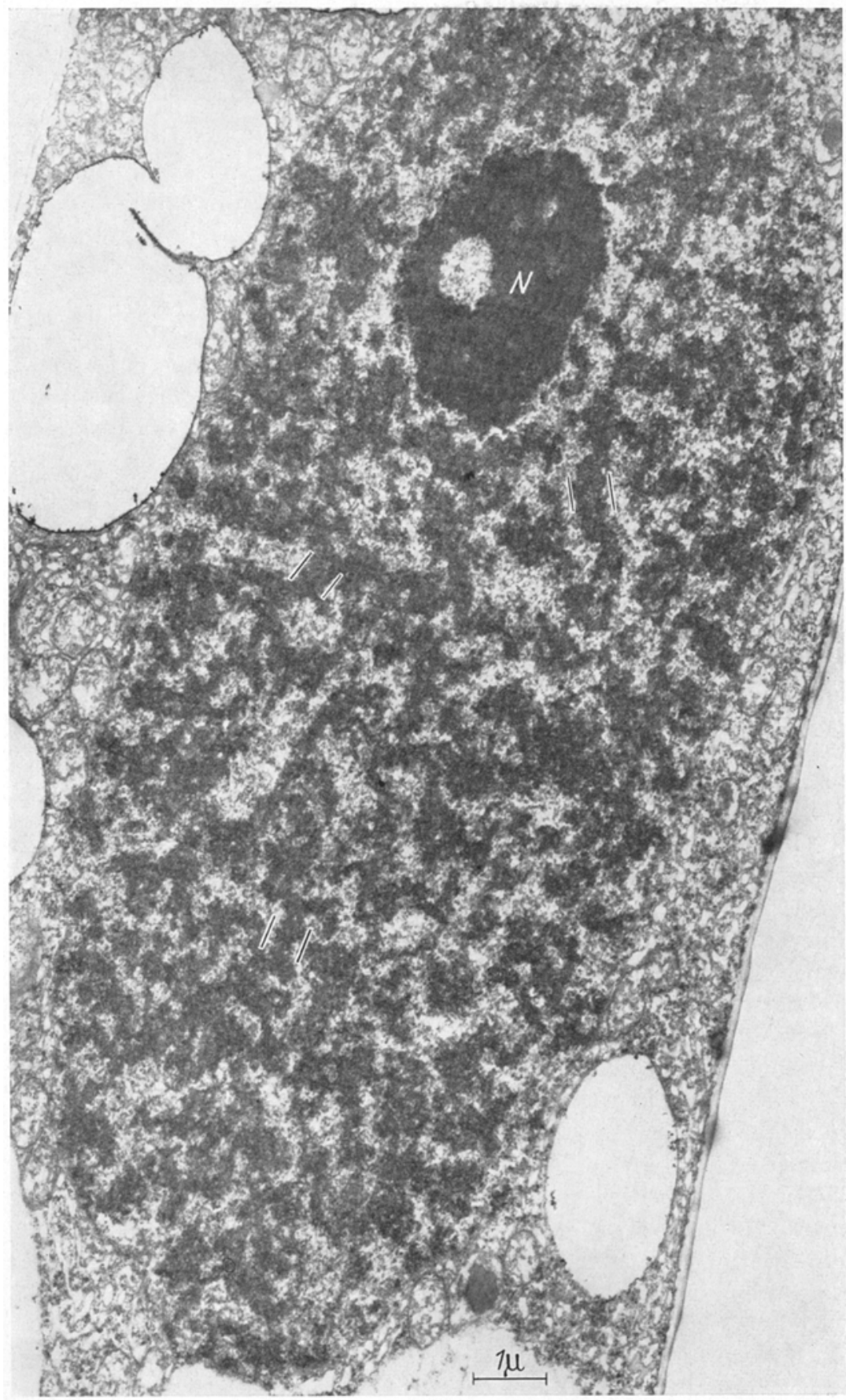

Fig. 13. Nucleus in middle interphase. On approaching preprophase the chromonematic material tends to aggregate again, suggestive in some places of the reorganization of chromonematic threads (double lines). Note the elongated shape of the nucleus. $(N)$ nucleolus. $\times 8,750$ 
lying in proximity to the nuclear membrane. Since these ten sections, each about $700 \AA$ thick, included more than half the diameter of the chromosome, it seems reasonable to conclude that the whole chromosome contains at least eight cytologically separable chromonemata. Another line of evidence in support of this interpretation is derived from analysis of the distribution of contours, sinuses, and open spaces in the models prepared by assembling large numbers of consecutive sections traversing an entire chromosome (e.g., chromosomes $C r 1$ and $C r$, Fig. 2).

In more familiar cytological terminology, each of the tetraspiremes constitutes a chromatid, which separates from its sister tetraspireme at anaphase to form one of the two daughter chromosomes that move toward the poles of the spindle. With respect to the manner of association of its constituent chromonemata, the metaphase chromosome appears to be fundamentally a pair of pairs of pairs (Fig. 5).

The pattern of chromonematic association that is evident during prophase is also apparent during telophase (Figs. 9, 10). As soon as the chromonemata become recognizable at telophase, it is possible to detect the diplospiremes. In early telophase, paired diplospiremes lie in close approximation, exhibiting the type of configuration that we ascribe to the association of pairs of double helices possessing opposite types of spiralization (Figs. 9, 10). In late telophase the close association is no longer apparent, but the existence of the constituent chromonemata is revealed by the free ends lying in contact with the nuclear membrane (Fig. 11).

Disappearance at metaphase of the chromonemata as individually discernible threads occurs concomitantly with the breakdown of the nuclear membrane. It is still possible in early metaphase to detect, however, the undulous contours of the chromosomes that assumedly reflect the spiral nature of their constituent chromonemata (Fig. 7). By early anaphase the chromosomes show an extraordinary degree of uniformity of densely-electron-scattering materials. This situation persists until late anaphase, but at that stage the chromosomes also appear to be sheathed in a dense material, as if they were shedding denselyelectron-scattering substances-ribosomal-like granules, filamentous and amorphous materials - as they move to the spindle poles (Fig. 8).

In the interphase nucleus, the positions of the chromonemata are marked by zones of dense material, but other fibrillar substances-having an affinity for uranyl acetate and appearing on the micrographs as dark-colored strands-are distributed throughout the nucleoplasm. (Fig. 12). The fate of these "extra-chromonematic nuclear materials" in the transition from interphase to pre-prophase remains to be determined, but the possibility exists that they become associated in some way with the more compact chromonemal "cores" to give rise to the definitive chromonemata (cf. Fig. 13). 


\section{Diseussion}

The interpretation given above, based on electron-microscopical evidence, that the anaphase-telophase chromosome is quadripartite with respect to the number of chromonemata lends support to the similar interpretation presented by NEBEL (1932) on the basis of studies with the light microscope. This, in turn, was grounded on the observation that paired chromonematic strands are constant chromosomal constituents at all phases of somatic and meiotic mitoses (KaUfMann, 1925, 1926). Viewed in historical perspective, it is interesting to note that the question of multiplicity of ehromonemata at anaphase and telophase has been challenged over the years on the basis of "logical inferences". During the period when the validity of the chromonemahypothesis was being debated, WILSON (1925) and KUWADA (1926) argued that "longitudinal splitting" of the chromosome in anaphase would mean a transversal (genetically disruptive) division at each turn of any existing spiral. And more recently, Taxuor (1957, 1958, 1959, $1962,1963)$ and others have invoked the logical inference that unanimity in functional capacity is indicative of singularity in structural organization. To do this has meant abandoning, by relegating to the eategory of artifactitious or aberrant, the extensive observational and experimental evidence that has been assembled during the past 40 years in favor of the view that chromosomes in higher plants and animals are multistranded (having two or more chromonemata) at all phases of somatic and meiotic mitoses.

The present study was undertaken to resolve the conflicting points of view by direct recourse to analysis of ehromonematic association at the electron-microscopical level. Although the interpretations given in this paper involve subjective evaluations of three-dimensional reconstructions, a measure of their validity is afforded by the following considerations. Tn the first place, the models prepared by stacking "wax impressions" of serial sections were reconstructed in modeling clay. These were then sliced at the different levels that corresponded to other selected electron micrographs. When this was done, the eut surface of the model frequently showed a close parallelism to the pattern of profiles and internal details seen on the micrograph. In the second place, the ability to detect and count the number of "separate chromonematic ends" of a prophase or telophase chromosome lying in contact with the nuclear membrane gave direct positive evidence that the chromosomes of $T$. paludosa are polynemic.

Eight chromonemata per chromosome have also been detected by HUGHES-SCHRADER (1940) in studies of meiosis in the male coccid, Llaveiella taenechina. The complex structure of the chromosomes of this coccid "is rendered dramatically visible by a unique autonomy of the individual chromatids in their metaphase behavior and their ana- 
phasic movements. Not only is the tertiary split present (the unused cleavage of the meiotic mitoses) but its reality is demonstrated beyond possibility of cytological misinterpretation by the fact that the pairs of half chromatids separated by it proceed in their poleward migration at individually different rates". "There is", HugHES-Schrader continues, "occasional scattered evidence for still another subdivision of the meiotic chromosome of Llaveiella". Nuclei in which this division is quite complete and in which the quarter chromatids differ in the rate of their poleward movements "offer ... conclusive evidence for the reality of the fourth split".

Attention has been directed to this classical illustration from coccid cytology because TAYLOR $(1963$, p. 67), in his efforts to present a unifying model of chromosome structure, has suggested that coccids, whose chromosomes have multiple or diffuse centromeres, may be exceptional (as compared with chromosomes of most animals and plants) in regard to the number of chromatids. The findings of the present study suggest that this is not the case. The exception appears to reside, not in the number of chromonemata, but in the manner of their coordination and integration to form aggregates that function as autonomous units. In Llaveiella separation of subunits is effected in the normal sequence of the meiotic process; in Tradescantia intimate association of constituent strands is normally maintained during the condensed phases of both somatic and meiotic mitoses. As noted in an earlier publication (KaUFManN, 1959), the discovery of subunits within a chromosome does not pose a new problem but does emphasize the need for a solution in terms of the conditions that can mold a group of strands into a functionally single aggregate.

It is noteworthy that both the light microscope and the electron microscope disclose similar patterns of chromosomal organization at the chromonematic level. Examination with each instrument reveals a structural unit composed of a pair of intertwined strands, which is integrated with other similar units to form a hierarchy of pairs of pairs. As would be expected in these cases, the end views of chromonemata appear at all levels of organization as rings or, in the case of our socalled diplospiremes, as ellipses.

In squashes of telophasic cells (e.g., MaNTon, 1945), the chromosomes appear as rope-like aggregates. Such appearances have been interpreted (e.g., KuWada, 1939; KaUfmann, 1948, KaUfmanN, Gay and McDonald 1960; RIs, 1961) as evidence of the existence of an aggregation of four chromonemata in two pairs of spirals. The present study leads to the same interpretation, but suggests in addition that the constituent pairs coil in opposite directions. Squashed metaphase or anaphase and, at times, prophase chromosomes often appear vacuolated 
along their lengths. This type of fixation artifact reflects, we believe, an enhancement of the normal lines of separation within the chromosome, probably as a result of the shrinkage and condensation of the materials that constitute the diplospiremes and tetraspiremes.

It is also possible to detect with both the light and the electron microscopes changes that occur in the chemical composition of chromosomes as they progress through the mitotic cycle. As had already been inferred from cytochemical studies with the light microscope, the anaphase to telophase transition is marked by the release from the chromosomes (at least in the plant and animal cells studied) of basic materials that appear to be ribonucleic acid or ribonucleoprotein (KAUFMANN, MCDonald, and GaY, 1948; Jacobson and WEBB, 1952). The electron micrographs assembled in the present study demonstrate at the fine structural level that ribosomal-like particles, as well as fibrillar and "amorphous" materials, are released from the chromonematic cores in the anaphase-telophase transition. The pattern of association of these materials within the chromosome and the manner of their release remain to be determined, but information along these lines should prove useful in correlating structural and functional aspects of chromosomal behavior.

The present study also leaves unanswered the question of subchromonematic organization. Electron micrographs of chromosomes in staminate-hair cells of Tradescantia disclose a wealth of fine fibrillae, each of which has a diameter of about $125 \AA$. KAUFMANN and DE (1956) concluded that these were the elementary subsidiary units, of which the prophase chromosome might contain as many as 64 . The validity of this interpretation must now be tested by the laborious construction of models from electron micrographs of serial sections in which fine detail can be resolved. Work along these lines is in progress. Preliminary studies suggest that radioautographic methods may be useful in the analysis. If so, we shall have the information that is essential for determining the distribution of isotopes among the several chromonemata (or sub-chromonemata) that constitute the chromosomes of higher plants and animals, and a firmer basis for assessing the validity of the various models of chromosome structure that have been proposed in recent years.

\section{Summary}

Analysis of reconstructions, prepared from electron micrographs of successive longitudinal serial sections, has led to the conclusion that the somatic telophase chromosome of Tradescantia paludosa contains four cytologically separable chromonemata. The four represent a pair of pairs, that is, two diplospiremes-one with its two chromonemata arranged helically in dextrorse relationship, and the other with its two in sinistrorse relationship - which are associated to form a tetra- 
spireme. During anaphase and telophase the tetraspireme constitutes the chromosome; during prophase and metaphase the tetraspireme represents one of the two chromatids of the chromosome, which is accordingly an octospireme in terms of the number of cytologically identifiable chromonemata. Loose intertwining of the two tetraspiremes during late prophase accounts for the so-called relational coiling.

\section{Literature}

CaUlfield, J. B.: Effects of varying the vehicle for $\mathrm{OsO}_{4}$ in tissue fixation. J. biophys. biochem. Cytol. 3, 827-829 (1957).

Freeman, J. A., and B. O. SpURLook: A new epoxy embedment for electron microscopy. J. Cell Biol. 13, 437-443 (1962).

GAY, H., and T. F. ANDERson: Serial sections for electron microscopy. Science 120, $1071-1073$ (1954).

HugHES-SCHRADER, S.: The meiotic chromosomes of the male Llaveiella taenechina Morrison (Coccidae) and the question of the tertiary split. Biol. Bull. 78, $312-337(1940)$.

Kaufmann, B. P.: The existence of double spiral chromatin bands and a "bouquet" stage in Tradescantia pilosa LeHm. Amer. Naturalist 59, 190 (1925); Chromosome structure and its relation to the chromosome cycle. Amer. J. Bot. 13, 59-80 (1926); - Chromosome structure in relation to the chromosome cycle. II. Bot. Rev. 14, 57-126 (1948); - Varying patterns of chromosomal fine strueture. In: The cell nucleus, p. 251-263. London: Butterworth \& Co. 1959.

一, and D. N. DE: Fine structure of chromosomes. J. biophys. biochem. Cytol., Suppl. 2, 419-424 (1956).

- H. GAY, and M. R. MoDonalD: Organizational patterns within chromosomes. Int. Rev. Cytol. 9, 77-127 (1960).

- M. R. MCDONALD, and H. GAY: Enzymatic degradation of ribonucleoproteins of chromosomes, nucleoli and cytoplasm. Nature (Lond.) 162, 814 (1948).

KUWADA, Y.: On the structure of the anaphasic chromosomes in the somatic mitosis in Vicia faba, with special reference to the so called longitudinal split of chromosomes in the telophase. Mem. Coll. Sci. Kyoto Imp. Univ., Ser. B 2, 1 -13 (1926); - Chromosome structure. Cytologia (Tokyo) 10, 213-256 (1939).

JACOBson, W., and M. WeBB: The two types of nucleoproteins during mitosis. Exp. Cell Res. 3, 163-183 (1952).

LA Cour, L. F., and S. R. Pelc: Effect of colchicine on the utilization of labeled thymidine during chromosomal reproduction. Nature (Lond.) 182, 506-508 (1958).

Manton, I. New evidence on the telophase split in Todea barbara. Amer. J. Bot. $32,342-348$ (1945).

NEBEL, B. R.: Chromosome structure in Tradescantiae I. Z. Zellforsch. 16, 251 to 284 (1932); - Chromosome structure. Bot. Rev. 5, 563-626 (1939).

Peacock, W. J.: Chromosome duplication and structure as determined by autoradiography. Proc. nat. Acad. Sci. (Wash.) 49, 793 - 801 (1963).

RIs, H.: Ultrastructure and molecular organization of genetic systems. Canad. J. Genet. Cytol. 8, 95-120 (1961).

Sparvoli, E., H. GaY, and B. P. Kadfmañ: Open face embedding of single cells for electron microscopy. Stain Technol, 40, 83-88 (1965). 
TAYLOR, J. H.: The time and mode of duplication of chromosomes. Amer. Naturalist 91, 209-221 (1957); - Sister chromatid exchanges in tritium labeled chromosomes. Genetics 43, 515-529 (1958); - The organization and duplication of genetic material. Proc. 10th Int. Congr. Genet. Montreal 1, 63-78 (1958); - Chromosome reproduction. Int. Rev. Cytol. 13, 39-73 (1962); Control mechanisms for chromosome reproduction in the cell cycle. In: Growth and division, p. 161-177, ed. by R. J. Harris. New York: Academic Press 1962; - The replication and organization of DNA in chromosomes. In: Molecular genetics, p. 65-111, ed. by J. H. TAYLOR. New York: Academic Press 1963.

TAYlor, J. H., P. S. Woods, and W. L. Hughes: The organization and duplication of chromosomes as revealed by autoradiographic studies using tritium labeled thymidine. Proc. nat. Acad. Sci. (Wash.) 43, 122-128 (1957).

WILSON, E. B.: The cell in development and heredity. New York: Macmillan 1925.

Dr. Elio Sparvoli

Department of Zoology, University of Michigan

Ann Arbor, Michigan 48104, USA 